\title{
Cosmic particle acceleration in astrophysical shear flows
}

\author{
Frank M. Rieger and Peter Duffy \\ UCD School of Mathematical Sciences, University College Dublin, Dublin 4, Ireland \\ email: frank.rieger,peter.duffy@ucd.ie
}

\begin{abstract}
Shear flows are ubiquitous phenomena in astrophysical environments. In the present contribution the stochastic acceleration of energetic charged particles by Fermi-type processes in relativistic shear flows is considered. We briefly summarize recent theoretical progress in the field of viscous shear acceleration and indicate its significance for particle energization in relativistic flows of AGNs and GRBs.
\end{abstract}

Keywords. acceleration of particles, galaxies: active, gamma rays: bursts

Relativistic shear flows are a natural outcome of density and velocity gradients in the extreme astrophysical environments around AGNs and GRBs and, at least in the case of AGNs, observationally well established (see, e.g., Rieger \& Duffy 2004 for review). We have shown elsewhere (see references) that scattering off magnetic turbulence structures embedded in such (collisionless) flows can lead to efficient Fermi-type particle acceleration and thus allow conversion of a non-negligible part of the bulk kinetic energy of the flow into nonthermal particles and radiation. For a momentum-dependent particle mean free path $\lambda(p) \propto p^{\alpha}$, acceleration of energetic particles in non-relativistic gradual shear flows is known to lead to (local) steady-state power-law particle distributions $n(p) \propto p^{-(1+\alpha)}$ above injection $p_{0}$ for $\alpha>0$. Hence, for a particle mean free path $\lambda \propto$ gyro-radius $r_{g} \propto p$, one has $n(p) \propto p^{-2}$. As particles sample a higher shear with increasing $\lambda$, i.e., experience a higher scattering impact, the characteristic acceleration timescale is inversely related to $\lambda$, i.e., one finds $t_{\text {acc }} \propto 1 / \lambda$ in marked contrast to shock-type acceleration processes (Rieger $\&$ Duffy 2006). In particular, for $\lambda \simeq r_{g}$ (Bohm case), acceleration $t_{\text {acc }}$ and radiative (synchrotron) cooling timescale $t_{\text {cool }}$ have the same scaling with $\gamma$, so that radiative losses are no longer able to balance acceleration once it has started to work efficiently. The results can be generalized to the relativistic case with a similar outcome (e.g., Rieger \& Duffy 2004). As particle energization appears possible as long as the shear persists, shear acceleration represents a natural candidate for distributed acceleration processes thought to be at work in large-scale relativistic AGN jets beyond the usual $2^{\text {nd }}$ order Fermi mechanism (Rieger et al. 2007). Moreover, efficient shear acceleration of protons up to UHE cosmic ray energies appears possible within the jets of AGNs and GRBs, in the latter case probably more efficiently than via internal shock-type processes (Rieger $\&$ Duffy 2005). Finally, in $\mu$ Quasar jets, shear acceleration may allow electron energies to reach those required to produce inverse Compton TeV emission (Rieger et al. 2007).

\section{References}

Rieger, F. M., \& Duffy, P. 2004, ApJ, 617, 155

Rieger, F. M., \& Duffy, P. 2005, ApJ (Letters), 632, L21

Rieger, F. M., \& Duffy, P. 2006, ApJ, 652, 1044

Rieger, F. M., Bosch-Ramon, V., \& Duffy, P. 2007, ApESSS, 309, 119 